\title{
On the stability of a non-Newtonian polytropic filtration equation
}

\author{
Huashui Zhan ${ }^{1 *}$ and Miao Ouyang
}

\section{"Correspondence:}

huashuizhan@163.com

${ }^{1}$ School of Applied Mathematics,

Xiamen University of Technology,

Xiamen, China

\begin{abstract}
The non-Newtonian polytropic filtration equation with a convection term

$$
v_{t}=\operatorname{div}\left(a(x)|v|^{\alpha}|\nabla v|^{p-2} \nabla v\right)+\sum_{i=1}^{N} \frac{\partial a_{i}(v, x, t)}{\partial x_{i}}
$$

is considered, where $p>1, \alpha>0, a(x) \geq 0$ with $\left.a(x)\right|_{x \in \partial \Omega}=0$. This kind of equation is degenerate on the boundary, the usual boundary value condition may be overdetermined. Some conditions depending on $a(x)$ and $a_{i}(\cdot, x, t)$, which can take place of the boundary value condition, are found. Moreover, how the nonlinear term $|v|^{\alpha}$ affects the stability of weak solutions is revealed.
\end{abstract}

MSC: 35K55; 35K92; 35K85; 35R35

Keywords: Non-Newtonian polytropic filtration equation; Convection term; Boundary value condition; Stability

\section{Introduction}

Consider non-Newtonian polytropic filtration equation with a convection term

$$
v_{t}=\operatorname{div}\left(a(x)|v|^{\alpha}|\nabla v|^{p-2} \nabla v\right)+\sum_{i=1}^{N} \frac{\partial a_{i}(v, x, t)}{\partial x_{i}}, \quad(x, t) \in \Omega \times(0, T),
$$

with the initial-boundary value conditions

$$
\begin{aligned}
& \left.v\right|_{t=0}=v_{0}(x), \quad x \in \Omega, \\
& v(x, t)=0, \quad(x, t) \in \partial \Omega \times(0, T),
\end{aligned}
$$

where $p>1, \alpha \geq 0, a(x) \in C^{1}(\bar{\Omega}), a_{i}(s, x, t) \in C^{1}(\mathbb{R} \times \bar{\Omega} \times[0, T])$ for every $i=1,2, \ldots, N$, $\Omega \subset \mathbb{R}^{N}$ is a bounded domain with a smooth boundary $\partial \Omega$. The equations like (1.1) arise from a variety of diffusion phenomena, such as soil physics, fluid dynamics, combustion theory and reaction chemistry, one can see [1-4] and the references therein. When $\alpha>$ $0, a(x) \equiv 1$, the well-posedness problem of Eq. (1.1) has been studied thoroughly. Many

(c) The Author(s) 2019. This article is distributed under the terms of the Creative Commons Attribution 4.0 International License (http://creativecommons.org/licenses/by/4.0/), which permits unrestricted use, distribution, and reproduction in any medium, provided you give appropriate credit to the original author(s) and the source, provide a link to the Creative Commons license, and indicate if changes were made. 
scholars had been interested in the polytropic infiltration equations of the form

$$
u_{t}=\operatorname{div}\left(|u|^{r}|\nabla u|^{p-2} \nabla u\right)+f(x, t, u, \nabla u),
$$

or equivalently

$$
u_{t}=\operatorname{div}\left(\left|\nabla u^{m}\right|^{p-2} \nabla u^{m}\right)+f(x, t, u, \nabla u) .
$$

To be more specific: If $f(x, t, u, \nabla u)=0$, the Cauchy problem of this kind of equation can be traced back to Gilding-Peletier [5]. Later, if the initial value $u_{0}(x) \in L^{1}\left(\mathbb{R}^{N}\right)$, the existence and the uniqueness of weak solution were studied and $u_{t} \in L^{1}\left(\mathbb{R}^{N} \times(\tau, T)\right)$ was proved for any $\tau>0$ [6]. If the initial value $u_{0}(x)$ is just a measure, a similar problem was considered in $[7,8]$. A more general equation was studied in [9] based on a $L^{1}$ initial value condition. If $f(x, t, u, \nabla u)$ is a source term, the Cauchy problem was studied in [10] etc. For the initialboundary value problem, when $f(x, t, u, \nabla u)=0$ and $u_{0}(x) \in L^{\infty}(\Omega)$, the well-posedness problem was studied in $[11,12]$ etc. When $f(x, t, u, \nabla u)=\nabla A(u)$, by modifying the usual Moser iteration and imposing some restrictions on the convection function $A(s)$, the local $L^{\infty}$-estimates were made and $u_{t} \in L^{2}\left(\mathbb{R}^{N} \times(\tau, T)\right)$ was proved, provided that $u_{0}(x) \in L^{q}(\Omega)$ with $q \geq 1$ [13]. The large time behavior of solutions had been studied in [14-16] etc. The extinction, positivity and the blow-up of solutions had been studied in $[17,18]$ etc. The finite speed propagation was studied in [19] provided that $f(x, t, u, \nabla u)=-\vec{\beta}(x) \cdot \nabla u^{q}$ is with orientated convection. Of course, there are a great deal of papers to study various subjects on these kinds of equations, for examples, one can refer to [20-26] and the references therein.

If we regard Eq. (1.1) as the generalization of the heat conduction equation, then $a(x)|v|^{\alpha}$ has the meaning of nonlinear thermal conductivity depending on the temperature $v=$ $v(x, t)$ [20]. If we consider Eq. (1.1) as the generalization of the reaction-diffusion equation, $a(x)$ is the diffusion coefficient [27]. When $a(x)=d(x)^{\beta}, \alpha=0$ and $a_{i} \equiv 0$ in Eq. (1.1), YinWang's work [28] reveals that the uniqueness of the weak solution is independent of the boundary value condition when $\beta>p-1$. Moreover, they generalized [27]'s work to the following equation:

$$
\frac{\partial u}{\partial t}-\operatorname{div}\left(a(x)|\nabla u|^{p-2} \nabla u\right)-\sum_{i=1}^{N} b_{i}(x) D_{i} u+c(x, t) u=f(x, t),
$$

where $a(x) \geq 0$, and divided the boundary $\partial \Omega$ into three parts: the non-degenerate part, the weakly degenerate part and the strongly degenerate part. On the first two parts, the boundary value condition can be imposed in the sense of the trace. While, in the strongly degenerate part, the boundary value condition only can be explained in a much weaker sense than the trace. Recently, using some ideas of [27], the existence and the uniqueness of weak solution to some special cases of Eq. (1.1) had been studied by the author in [2931 , where $a(x)$ satisfies

$$
a(x)>0, \quad x \in \Omega, \quad a(x)=0, \quad x \in \partial \Omega .
$$


We have found that the Dirichlet boundary condition (1.3) may be overdetermined. Instead, a partial boundary value condition

$$
v(x, t)=0, \quad(x, t) \in \Sigma_{p} \times(0, T),
$$

is required when the stability of weak solutions is discussed, where $\Sigma_{p} \subseteq \partial \Omega$ is a relatively open subset of $\partial \Omega$. The problem lies in that, by choosing different test functions, we only can find the different $\Sigma_{p} \subset \partial \Omega$ in (1.5). From this we observe that how to find an exact partial boundary $\Sigma_{p}$ in (1.5) to match up with the nonlinear equations with the type (1.1) seems almost impossible. In this paper, we may ponder about and tackle the problem from another perspective. We are ready to find some conditions, which are related to $a(x)$ and $a_{i}(\cdot, x, t)$, to take place of the boundary value condition (1.3) (or (1.5)). Technically, since $\alpha>$ 0 , how the nonlinear term $|v|^{\alpha}$ affects the stability of weak solutions is the main difficulty we should overcome. Let us give the definition of weak solution.

Definition 1.1 A function $u(x, t)$ is said to be a weak solution of Eq. (1.1) with the initial value (1.2), if

$$
v \in L^{\infty}\left(Q_{T}\right), \quad \frac{\partial v}{\partial t} \in L^{p^{\prime}}\left(0, T ; W^{1, p^{\prime}}(\Omega)\right), \quad a(x)|v|^{\alpha}|\nabla v|^{p} \in L^{1}\left(Q_{T}\right),
$$

and, for any function $\varphi \in C_{0}^{1}\left(Q_{T}\right)$,

$$
\iint_{Q_{T}}\left(\frac{\partial v}{\partial t} \varphi+a(x)|v|^{\alpha}|\nabla v|^{p-2} \nabla v \cdot \nabla \varphi+\sum_{i=1}^{N} a_{i}(v, x, t) \varphi_{x_{i}}\right) d x d t=0 .
$$

The initial value (1.2) is satisfied in the sense of

$$
\lim _{t \rightarrow 0} \int_{\Omega}\left|v(x, t)-v_{0}(x)\right| d x=0
$$

If $v$ satisfies the boundary value condition (1.3) (or the partial boundary value condition (1.5)) in the sense of the trace, then we say $v$ is a weak solution of the initial-boundary value problem of Eq. (1.1).

Here $p^{\prime}=\frac{p}{p-1}$. By the parabolically regularized method, we can prove the following existence theorem.

Theorem 1.2 If $p \geq 2, a(x) \in C^{1}(\bar{\Omega})$ satisfies (1.4), $a_{i}(s, x, t) \in C^{1}(\mathbb{R} \times \bar{\Omega} \times[0, T])$,

$$
\begin{aligned}
& \frac{\partial a_{i}}{\partial x_{i}} \leq 0, \\
& 0 \leq v_{0} \in L^{\infty}(\Omega), \quad v_{0} \in W^{1, p}(\Omega),
\end{aligned}
$$

then Eq. (1.1) with the initial value (1.2) has a nonnegative solution v. Moreover, if we add the additional condition

$$
\int_{\Omega} a(x)^{-\delta} d x<\infty
$$


where $\delta>0$ satisfied $\frac{1}{\delta} \leq p-1$, then the boundary value condition (1.3) (or the partial boundary value condition (1.5)) is true in the sense of the trace.

We do not pay so much attention on the existence of the weak solution. Theorem 1.2 is given for the completeness of the paper. Actually, when $a_{i} \equiv 0, i=1,2, \ldots, N$, only if $p>1$, the existence of weak solution with $\frac{\partial v}{\partial t} \in L^{2}\left(Q_{T}\right)$ had been proved in [30]. At the same time, the existence of weak solution to Eq. (1.1) can be proved in the other sense [31]. Moreover, the condition $v_{0} \in W^{1, p}(\Omega)$ can be weaken to $a(x)\left|\nabla v_{0}\right|^{p} \in L^{1}(\Omega)$. In addition, if $1<p \leq 2$ and $a_{i}(v, x, t) \equiv 0$, how the degeneracy of $a(x)$ on the boundary affects the stability of weak solutions had first been studied in [30]. If $a_{i}(v, x, t)=a_{i}(v)$, the stability of a class of solutions satisfying

$$
\frac{1}{\lambda}\left(\int_{\Omega \backslash \Omega_{\lambda}} a(x)\left|\nabla v^{m}\right|^{p} d x\right)^{\frac{1}{p^{\prime}}} \leq c,
$$

had been originally studied in [31], where $m>1$ and $\Omega_{\lambda}=\{x \in \Omega: a(x)>\lambda\}$. Different from the method used in $[30,31]$, in order to prove the stability of weak solutions, we transfer Eq. (1.1) to another type of equation. Let $v=|u|^{\beta-1} u, \beta=\frac{(p-1)}{(\alpha+p-1)}, \delta=\beta^{p-1}$. Then the main equation (1.1) becomes

$$
\left(|u|^{\beta-1} u\right)_{t}=\delta \operatorname{div}\left(a(x)|\nabla u|^{p-2} \nabla u\right)+\sum_{i=1}^{N} \frac{\partial b_{i}(u, x, t)}{\partial x_{i}}, \quad(x, t) \in \Omega \times(0, T),
$$

and with the usual initial-boundary value conditions

$$
\begin{aligned}
& u(x, 0)=u_{0}(x), \quad x \in \Omega, \\
& u(x, t)=0, \quad(x, t) \in \partial \Omega \times(0, T),
\end{aligned}
$$

where $u_{0}(x)=\left|v_{0}(x)\right|^{-1+\frac{1}{\beta}} v_{0}(x), b_{i}(u, x, t)=a_{i}\left(|u|^{\beta-1} u, x, t\right)$.

A function $u(x, t)$ is said to be a weak solution of Eq. (1.9) by that

$$
u \in L^{\infty}\left(Q_{T}\right), \quad \frac{\partial|u|^{\beta-1} u}{\partial t} \in L^{p^{\prime}}\left(0, T ; W^{1, p^{\prime}}(\Omega)\right), \quad a(x)|\nabla u|^{p} \in L^{1}\left(Q_{T}\right) .
$$

A basic result of the stability is the following.

Theorem 1.3 Let a(x) satisfy (1.4), $u(x, t)$ and $v(x, t)$ be two solutions of the initialboundary value problem (1.9)-(1.11). If

$$
\int_{\Omega} a(x)^{-\frac{1}{p-1}} d x<\infty
$$

then

$$
\left.\int_{\Omega}|| u\right|^{\beta-1} u-|v|^{\beta-1} v\left|d x \leq \int_{\Omega}\right|\left|u_{0}\right|^{\beta-1} u_{0}(x)-\left|v_{0}\right|^{\beta-1} v_{0}(x) \mid d x .
$$


Roughly speaking, if $u(x, t)$ is a weak solution of Eq. (1.9) with the initial value (1.10), if condition (1.12) is true, then $\int_{\Omega}|\nabla u| d x<\infty$, and the boundary value condition (1.11) can be imposed in the sense of the trace. Accordingly, similar as the usual evolutionary $p$-Laplacian equation, we can prove Theorem 1.3.

The main aim of this paper is to find the other conditions to replace the boundary value condition (1.11) (or equivalently, (1.3)). At first, even condition (1.12) is true and one can impose the boundary value condition (1.11), we still hope the stability of weak solutions can be obtained without the boundary value condition (1.11).

Theorem 1.4 Let a $(x)$ satisfy (1.4), $u(x, t)$ and $v(x, t)$ be two solutions of Eq. (1.9) with the initial values $u_{0}(x), v_{0}(x)$, respectively, but without any boundary value condition. If

$$
\frac{1}{\lambda}\left(\lambda \int_{\Omega \backslash \Omega_{\lambda}}|\nabla a|^{p} d x\right)^{\frac{1}{p}} \leq c
$$

and either

$$
|\nabla a|=0, \quad x \in \partial \Omega
$$

or

$$
\int_{\Omega} a(x)^{-\frac{1}{p-1}} d x<\infty, \quad \text { and } \quad b_{i}(\cdot, x, t)=0, \quad x \in \partial \Omega
$$

then the stability of the weak solutions is true in the sense of (1.13).

If there is not condition (1.12), then one cannot impose the boundary value condition (1.11) generally. In this case, there is only way to go. We have to try to prove the stability of weak solutions without any boundary value condition. The following theorem partially achieves our goals.

Theorem 1.5 Let $u(x, t), v(x, t)$ be two weak nonnegative solutions of Eq. (1.1) with the initial values $u_{0}(x), v_{0}(x)$, respectively. If a $(x)$ satisfies (1.4), and

$$
\int_{\Omega} a(x)^{-(p-1)} d x<\infty
$$

for every $i$,

$$
\left|a_{i}(u, x, t)-a_{i}(v, x, t)\right| \leq c a(x)^{\frac{1}{p}}\left|u^{m}-v^{m}\right|
$$

and there is a function $g_{i}(x)$ such that

$$
\begin{aligned}
& \left|a_{i}(\cdot, x, t)\right| \leq c g_{i}(x), \\
& \int_{\Omega} \frac{|\nabla a|}{a} g^{i}(x) d x \leq c,
\end{aligned}
$$


then the stability is true in the sense of that

$$
\int_{\Omega}|u(x, t)-v(x, t)| d x \leq c \int_{\Omega}\left|u_{0}(x)-v_{0}(x)\right| d x, \quad t \in[0, T) .
$$

One can see that the condition $\int_{\Omega} a(x)^{-\frac{1}{p-1}} d x<\infty$ and the condition $\int_{\Omega} a(x)^{-(p-1)} d x<$ $\infty$ cannot be true at the same time unless $p=2$. This fact shows that Theorem 1.4 and Theorem 1.5 complement each other.

At last, since the nonlinear convection term $\sum_{i=1}^{N} \frac{\partial a_{i}(v, x, t)}{\partial x_{i}}$ depends on the spatial variable $x$, we will use some ideas in [29-31] to prove the stability of weak solutions based on the partial boundary value condition.

Theorem 1.6 Let $u(x, t)$ and $v(x, t)$ be two solutions of Eq. (1.9) with the initial values $u_{0}(x)$, $v_{0}(x)$, respectively. If $a(x)$ satisfies (1.4), and there is a function $h_{i}(x)$ such that

$$
\left|b_{i}(\cdot, x, t)\right| \leq c h_{i}(x), \quad i=1,2, \ldots, N
$$

the partial boundary value condition

$$
u(x, t)=v(x, t)=0, \quad x \in \Sigma_{p}=\left\{x \in \partial \Omega: \sum_{i=1}^{N} h_{i}(x) a_{x_{i}} \neq 0\right\},
$$

is imposed, then the stability of the weak solutions is true in the sense of (1.13).

Here, we give a simple comment on Theorem 1.6. One can find that condition (1.12) does not appear in this theorem. But, in general, under condition (1.12), $\int_{\Omega}|\nabla u| d x<\infty$ is true, and the partial boundary value condition (1.23) can be imposed in the sense of the trace. Accordingly, we can say that condition (1.12) is hidden in condition (1.23). However, as we have said before, we cannot judge whether the partial boundary value condition (1.23) is the optimal one for Eq. (1.9) or not.

\section{Existence of the weak solutions}

The existence of weak solutions can be obtained by the parabolically regularized method. Consider the approximate problem

$$
\begin{aligned}
& u_{\varepsilon t}-\varepsilon \operatorname{div}\left(\left|\nabla u_{\varepsilon}\right|^{p-2} \nabla u_{\varepsilon}\right)-\operatorname{div}\left(a(x)\left|u_{\varepsilon}\right|^{\alpha}\left|\nabla u_{\varepsilon}\right|^{p-2} \nabla u_{\varepsilon}\right)-\sum_{i=1}^{N} \frac{\partial a_{i}\left(u_{\varepsilon}, x, t\right)}{\partial x_{i}} \\
& \quad=0, \quad(x, t) \in \Omega \times(0, T), \\
& u_{\varepsilon}(x, t)=0, \quad(x, t) \in \partial \Omega \times(0, T), \\
& u_{\varepsilon}(x, 0)=u_{\varepsilon 0}(x), \quad x \in \Omega .
\end{aligned}
$$

Here, $0 \leq u_{\varepsilon 0} \in C_{0}^{\infty}(\Omega),\left\|u_{\varepsilon 0}\right\|_{L^{\infty}(\Omega)} \leq\left\|u_{0}\right\|_{L^{\infty}(\Omega)}, u_{\varepsilon 0} \rightarrow u_{0}(x)$ in $W^{1, p}(\Omega)$. It is well-known that the above problem has a unique nonnegative solution $u_{\varepsilon} \in L^{\infty}\left(0, T ; W_{0}^{1, p}(\Omega)\right)$ [20], provided that $\frac{\partial a_{i}}{\partial x_{i}} \leq 0$. 
Proof of Theorem 1.2 By the maximum principle [20],

$$
\left\|u_{\varepsilon}\right\|_{L^{\infty}\left(Q_{T}\right)} \leq c
$$

here and the after $Q_{T}=\Omega \times(0, T)$.

Multiplying (2.1) by $u_{\varepsilon}$, by (2.4) and $a_{i}(s, x, t) \in C^{1}(\mathbb{R} \times \bar{\Omega} \times[0, T])$, since

$$
\begin{aligned}
\int_{\Omega} \frac{\partial a_{i}\left(u_{\varepsilon}, x, t\right)}{\partial x_{i}} u_{\varepsilon} d x & =-\int_{\Omega} a_{i}\left(u_{\varepsilon}, x, t\right) u_{\varepsilon x_{i}} d x \\
& =-\int_{\Omega} \frac{\partial}{\partial x_{i}} \int_{0}^{u_{\varepsilon}} a_{i}(s, x, t) d s d x+\int_{\Omega} \int_{0}^{u_{\varepsilon}} \frac{\partial}{\partial x_{i}} a_{i}(s, x, t) d s d x \\
& =\int_{\Omega} \int_{0}^{u_{\varepsilon}} \frac{\partial}{\partial x_{i}} a_{i}(s, x, t) d s d x
\end{aligned}
$$

using the equality

$$
\begin{gathered}
\frac{1}{2} \int_{\Omega} u_{\varepsilon}^{2} d x+\varepsilon \iint_{Q_{T}}\left|\nabla u_{\varepsilon}\right|^{p} d x d t+\iint_{Q_{T}} a(x)\left|u_{\varepsilon}\right|^{\alpha}\left|\nabla u_{\varepsilon}\right|^{p} d x d t \\
=\frac{1}{2} \int_{\Omega} u_{0}^{2} d x+\sum_{i=1}^{N} \iint_{Q_{T}} a_{i}\left(u_{\varepsilon}, x, t\right) u_{\varepsilon x_{i}} d x d t \\
=\frac{1}{2} \int_{\Omega} u_{0}^{2} d x+\sum_{i=1}^{N} \int_{\Omega} \int_{0}^{u_{\varepsilon}} \frac{\partial}{\partial x_{i}} a_{i}(s, x, t) d s d x,
\end{gathered}
$$

and by $a_{i}(s, x, t) \in C^{1}(\mathbb{R} \times \bar{\Omega} \times[0, T])$ and (2.4), one has

$$
\int_{\Omega} u_{\varepsilon}^{2} d x+\varepsilon \iint_{Q_{T}}\left|\nabla u_{\varepsilon}\right|^{p} d x d t+\iint_{Q_{T}} a(x)\left|u_{\varepsilon}\right|^{\alpha}\left|\nabla u_{\varepsilon}\right|^{p} d x d t \leq c
$$

which implies

$$
\begin{aligned}
& \iint_{Q_{T}} a(x)\left|u_{\varepsilon}\right|^{\alpha}\left|\nabla u_{\varepsilon}\right|^{p} d x d t \leq c, \\
& \varepsilon \iint_{Q_{T}}\left|\nabla u_{\varepsilon}\right|^{p} \leq c .
\end{aligned}
$$

For any $v \in L^{p}\left(0, T ; W_{0}^{1, p}(\Omega)\right),\|v\|_{L^{p}\left(0, T ; W_{0}^{1, p}(\Omega)\right)}=1$, using Young's inequality, one has

$$
\begin{aligned}
& \left.\left|\iint_{Q_{T}} a(x)\right| u_{\varepsilon}\right|^{\alpha}\left|\nabla u_{\varepsilon}\right|^{p-2} \nabla u_{\varepsilon} \nabla v d x d t \mid \\
& \quad \leq c \iint_{Q_{T}} a(x)\left|u_{\varepsilon}\right|^{\alpha}\left(\left|\nabla u_{\varepsilon}\right|^{p}+|\nabla v|^{p}\right) d x d t \\
& \quad \leq c \iint_{Q_{T}} a(x)\left|u_{\varepsilon}\right|^{\alpha}\left|\nabla u_{\varepsilon}\right|^{p} d x d t+c \\
& \quad \leq c,
\end{aligned}
$$


and since $a_{i}(s, x, t) \in C^{1}(\mathbb{R} \times \bar{\Omega} \times[0, T]),\left|u_{\varepsilon}\right| \leq c$, one has

$$
\left|\iint_{Q_{T}} a_{i}\left(u_{\varepsilon}, x, t\right) v_{x_{i}} d x d t\right| \leq c+c\|v\|_{L^{p}\left(0, T ; W_{0}^{1, p}(\Omega)\right)} \leq c .
$$

Then

$$
\left|\left\langle u_{\varepsilon}, v\right\rangle\right| \leq c\left[\varepsilon \iint_{Q_{T}}\left|\nabla u_{\varepsilon}\right|^{p} d x d t+\iint_{Q_{T}} a(x)\left|u_{\varepsilon}\right|^{\alpha}\left|\nabla u_{\varepsilon}\right|^{p} d x d t+1\right] \leq c
$$

and

$$
\left\|u_{\varepsilon t}\right\|_{L^{p^{\prime}}\left(0, T ; W^{-1, p^{\prime}(\Omega)}\right.} \leq c
$$

Certainly, for any $\varphi \in C_{0}^{1}(\Omega), 0 \leq \varphi \leq 1$, one has

$$
\left\|\left(\varphi u_{\varepsilon}\right)_{t}\right\|_{L^{p^{\prime}}\left(0, T ; W^{\left.-1, p^{\prime}(\Omega)\right)}\right.} \leq c,
$$

which yields

$$
\left\|\left(\varphi u_{\varepsilon}^{m}\right)_{t}\right\|_{L^{p^{\prime}}\left(0, T ; W^{-1, p^{\prime}}(\Omega)\right)} \leq c .
$$

Here and afterwards, $m=1+\frac{\alpha}{p-1}$.

At the same time, since $a(x) \in C^{1}(\bar{\Omega})$ and $\left.a(x)\right|_{x \in \Omega}>0$, (2.6) yields

$$
\left\|\varphi u_{\varepsilon}^{m}\right\|_{L^{p^{\prime}}\left(0, T ; W_{0}^{1, p}(\Omega)\right)} \leq c .
$$

For a fixed $s$ such that $s>\frac{N}{2}+1$, one has $H_{0}^{s}(\Omega) \hookrightarrow W^{1, p}(\Omega)$. Consequently, $W^{-1, p^{\prime}}(\Omega) \hookrightarrow$ $H^{-s}(\Omega)$. As a result, one has

$$
\left\|\left(\varphi u_{\varepsilon}^{m}\right)_{t}\right\|_{L^{p^{\prime}}\left(0, T ; H^{-s}(\Omega)\right)} \leq c .
$$

Let $u_{1 \varepsilon}=u_{\varepsilon}^{m}$. Then, by (2.12)-(2.13), one has

$$
\begin{gathered}
\left\|\varphi u_{1 \varepsilon}\right\|_{L^{p^{\prime}}\left(0, T ; W_{0}^{1, p}(\Omega)\right)} \leq c, \\
\left\|\left(\varphi u_{1 \varepsilon}\right)_{t}\right\|_{L^{p^{\prime}}\left(0, T ; H^{-s}(\Omega)\right)} \leq c .
\end{gathered}
$$

Noticing that $W_{0}^{1, p}(\Omega) \hookrightarrow L^{p}(\Omega) \hookrightarrow H^{-s}(\Omega)$, one can employ Aubin's compactness theorem in [32] to obtain $\varphi u_{1 \varepsilon} \rightarrow \varphi u_{1}$ strongly in $L^{p^{\prime}}\left(0, T ; L^{p}(\Omega)\right)$. Thus $\varphi u_{1 \varepsilon} \rightarrow \varphi u_{1}$ a.e. in $Q_{T}$. In particular, due to the arbitraries of $\varphi, u_{1 \varepsilon} \rightarrow u_{1}$ a.e. in $Q_{T}$. Accordingly, $u_{\varepsilon} \rightarrow u$ a.e. in $Q_{T}$.

Now, by (2.4) and (2.7), one has

$$
u \in L^{\infty}\left(Q_{T}\right)
$$

and

$$
\varepsilon\left|\nabla u_{\varepsilon}\right|^{p-2} \nabla u_{\varepsilon} \rightarrow 0, \quad \text { in } L^{\frac{p}{p-1}}\left(Q_{T}\right),
$$




$$
\begin{aligned}
& u_{\varepsilon} \rightarrow * u, \quad \text { in } L^{\infty}\left(Q_{T}\right), \\
& a_{i}\left(u_{\varepsilon}, x, t\right) \rightarrow a_{i}(u, x, t), \quad \text { a.e. in } Q_{T} .
\end{aligned}
$$

Moreover, by (2.6), there is a $n$-dimensional vector function $\vec{\zeta}=\left(\zeta_{1}, \ldots, \zeta_{n}\right),\left|\zeta_{i}\right| \in$ $L^{\frac{p}{p-1}}\left(Q_{T}\right)$, such that

$$
a(x)\left|u_{\varepsilon}\right|^{\alpha}\left|\nabla u_{\varepsilon}\right|^{p-2} \nabla u_{\varepsilon}=\frac{a(x)}{m^{p-1}}\left|\nabla u_{\varepsilon}^{m}\right|^{p-2} \nabla u^{m} \rightarrow \zeta, \quad \text { in } L^{\frac{p}{p-1}}\left(Q_{T}\right) .
$$

At last, by a similar $p$-Laplacian to the usual evolutionary one, it is not difficult to prove that

$$
\begin{aligned}
& \iint_{Q_{T}} a(x)|u|^{\alpha}|\nabla u|^{p-2} \nabla u \cdot \nabla \varphi d x d t \\
& \quad=\iint_{Q_{T}} \frac{a(x)}{m^{p-1}}\left|\nabla u^{m}\right|^{p-2} \nabla u^{m} \cdot \nabla \varphi d x d t \\
& \quad=\iint_{Q_{T}} \vec{\zeta} \cdot \nabla \varphi d x d t,
\end{aligned}
$$

for any given function $\varphi \in C_{0}^{1}\left(Q_{T}\right)$. One can refer to [33] for the details. Also by [33], the initial value (1.2) can be proved in the sense of (1.7). Then $u$ is a nonnegative weak solution of Eq. (1.1) with the initial value (1.2).

Lemma 2.1 ([30]) Let $u$ be a solution of Eq. (1.1) with the initial value (1.2). For the constants $s, \beta$ satisfying $s>\alpha \delta+1, \frac{1}{\delta} \leq p-1$, such that $\int_{\Omega} a^{-\delta}(x) d x<\infty$, we have

$$
\int_{Q_{T}}\left|\nabla u^{s}\right| d x d t \leq c
$$

By Lemma 2.1, the conclusion of Theorem 1.2 follows easily.

\section{The Proof of Theorem 1.3}

Lemma 3.1 Let $u_{t} \in L^{p^{\prime}}\left(0, T ; W^{-1, p^{\prime}}(\Omega)\right)$. For any continuous function $h(s), H(s)=$ $\int_{0}^{s} h(s) d s$, a.e. $t_{1}, t_{2} \in(0, T)$,

$$
\int_{t_{1}}^{t_{2}} \int_{\Omega} h(u) u_{t} d x d t=\left[\int_{\Omega}\left(H(u)\left(x, t_{2}\right)-H(u)\left(x, t_{1}\right)\right) d x\right]
$$

This is a minor generalized result of Lemma 2.2 in [34].

Proof of Theorem 1.3 If $u(x, t), v(x, t)$ are two nonnegative solutions of Eq. (1.9) with the same homogeneous boundary value and with the different initial values $u_{0}(x), v_{0}(x)$, respectively.

From the definition of the weak solution, for $\varphi \in L^{\infty}\left(0, T ; W_{0}^{1, p}(\Omega)\right)$, one has

$$
\begin{aligned}
\int_{\Omega} \varphi & \frac{\partial\left(|u|^{\beta-1} u-|v|^{\beta-1} v\right)}{\partial t} d x \\
& +\delta \int_{\Omega} a(x)\left(|\nabla u|^{p-2} \nabla u-|\nabla v|^{p-2} \nabla v\right) \cdot \nabla \varphi d x
\end{aligned}
$$




$$
\begin{aligned}
& +\int_{\Omega}\left[b_{i}(u, x, t)-b_{i}(v, x, t)\right] \varphi_{x_{i}} d x \\
= & 0 .
\end{aligned}
$$

For small $\eta>0$, let

$$
S_{\eta}(s)=\int_{0}^{s} h_{\eta}(\tau) d \tau, \quad h_{\eta}(s)=\frac{2}{\eta}\left(1-\frac{|s|}{\eta}\right)_{+} .
$$

Obviously $h_{\eta}(s) \in C(\mathbb{R})$, and

$$
\left|S_{\eta}(s)\right| \leq 1 ; \quad \lim _{\eta \rightarrow 0} S_{\eta}(s)=\operatorname{sign} s, \quad \lim _{\eta \rightarrow 0} S_{\eta}^{\prime}(s)=0 .
$$

Since $u(x, t)$ and $v(x, t)$ have the same homogeneous boundary value condition, by taking the limit, one can choose $\varphi=S_{\eta}(u-v)$ as the test function, then

$$
\begin{aligned}
\int_{\Omega} & S_{\eta}(u-v) \frac{\partial\left(|u|^{\beta-1} u-|v|^{\beta-1} v\right)}{\partial t} d x \\
& +\delta \int_{\Omega} a(x)\left(|\nabla u|^{p-2} \nabla u-|\nabla v|^{p-2} \nabla v\right) \cdot \nabla(u-v) S_{\eta}^{\prime}(u-v) d x \\
= & -\int_{\Omega}\left[b_{i}(u, x, t)-b_{i}(v, x, t)\right](u-v)_{x_{i}} S_{\eta}^{\prime}(u-v) d x .
\end{aligned}
$$

In the first place, by Lemma 3.1, one has

$$
\begin{aligned}
& \lim _{\eta \rightarrow 0} \int_{\Omega} S_{\eta}(u-v) \frac{\partial\left(|u|^{\beta-1} u-|v|^{\beta-1} v\right)}{\partial t} \mathrm{~d} x \\
& \quad=\int_{\Omega} \operatorname{Sign}(u-v) \frac{\partial\left(|u|^{\beta-1} u-|v|^{\beta-1} v\right)}{\partial t} \mathrm{~d} x \\
& \quad=\int_{\Omega} \operatorname{Sign}\left(|u|^{\beta-1} u-|v|^{\beta-1} v\right) \frac{\partial\left(|u|^{\beta-1} u-|v|^{\beta-1} v\right)}{\partial t} \mathrm{~d} x \\
& \quad=\left.\frac{d}{d t} \int_{\Omega}|| u\right|^{\beta-1} u-|v|^{\beta-1} v \mid d x .
\end{aligned}
$$

In the second place,

$$
\int_{\Omega} a(x)\left(|\nabla u|^{p-2} \nabla u-|\nabla v|^{p-2} \nabla v\right) \cdot \nabla(u-v) S_{\eta}^{\prime}(u-v) \mathrm{d} x \geq 0 .
$$

Last but not least, by (1.12), using the Lebesgue dominated convergence theorem, from (3.2), one has

$$
\begin{aligned}
& \lim _{\eta \rightarrow 0}\left|\int_{\Omega}\left[b_{i}(u, x, t)-b_{i}(v, x, t)\right](u-v)_{x_{i}} S_{\eta}^{\prime}(u-v) d x\right| \\
& \leq \lim _{\eta \rightarrow 0}\left(\int_{\Omega}\left|\left[b_{i}(u, x, t)-b_{i}(v, x, t)\right] S_{\eta}^{\prime}(u-v) a^{-\frac{1}{p}}\right|^{\frac{p}{p-1}} d x\right)^{\frac{p-1}{p}} \\
& \cdot\left(\int_{\Omega} a(x)\left(|\nabla u|^{p}+|\nabla v|^{p}\right) d x\right)^{\frac{1}{p}}=0 .
\end{aligned}
$$


At last, let $\eta \rightarrow 0$ in (3.3). By (3.4)-(3.6), one has

$$
\frac{d}{d t}\left\||u|^{\beta-1} u-|v|^{\beta-1} v\right\|_{L^{1}(\Omega)} \leq 0 .
$$

It implies that

$$
\left.\int_{\Omega}|| u\right|^{\beta-1} u-|v|^{\beta-1} v\left|\mathrm{~d} x \leq \int_{\Omega}\right|\left|u_{0}\right|^{\beta-1} u_{0}-\left|v_{0}\right|^{\beta-1} v_{0} \mid \mathrm{d} x, \quad \forall t \in[0, T) .
$$

\section{The Proofs of Theorem 1.4 and Theorem 1.6}

Proof of Theorem 1.4 For a small positive constant $\lambda>0$, let

$$
\Omega_{\lambda}=\{x \in \Omega: a(x)>\lambda\}
$$

as before, and

$$
\phi_{\lambda}(x)= \begin{cases}1, & \text { if } x \in \Omega_{\lambda} \\ \frac{a(x)}{\lambda}, & x \in \Omega \backslash \Omega_{\lambda} .\end{cases}
$$

Now, by taking the limit, one can choose $\varphi=\phi_{\lambda}(x) \chi_{[\tau, s]} S_{\eta}(u-v)$, and integrate it over $Q_{T}$, one has

$$
\begin{aligned}
& \int_{\tau}^{s} \int_{\Omega} \phi_{\lambda}(x) S_{\eta}(u-v) \frac{\partial\left(|u|^{\beta-1} u-|v|^{\beta-1} v\right)}{\partial t} d x d t \\
& \quad+\delta \int_{\tau}^{s} \int_{\Omega} \phi_{\lambda}(x) a(x)\left(|\nabla u|^{p-2} \nabla u-|\nabla v|^{p-2} \nabla v\right) \cdot \nabla(u-v) S_{\eta}^{\prime}(u-v) d x d t \\
& \quad+\delta \int_{\tau}^{s} \int_{\Omega} a(x)\left(|\nabla u|^{p-2} \nabla u-|\nabla v|^{p-2} \nabla v\right) \cdot \nabla \phi_{\lambda}(x) S_{\eta}(u-v) d x d t \\
& \quad+\int_{\tau}^{s} \int_{\Omega}\left[b_{i}(u, x, t)-b_{i}(v, x, t)\right]\left[\phi_{\lambda}(x) S_{\eta}^{\prime}(u-v)(u-v)_{x_{i}}+S_{\eta}(u-v) \phi_{\lambda x_{i}}(x)\right] d x d t \\
& =0
\end{aligned}
$$

At first, one has

$$
\int_{\Omega} \phi_{\lambda}(x) a(x)\left(|\nabla u|^{p-2} \nabla u-|\nabla v|^{p-2} \nabla v\right) \cdot \nabla(u-v) S_{\eta}^{\prime}(u-v) d x \geq 0
$$

and

$$
\begin{aligned}
& \left|\int_{\Omega} a(x)\left(|\nabla u|^{p-2} \nabla u-|\nabla v|^{p-2} \nabla v\right) \cdot \nabla \phi_{\lambda}(x) S_{\eta}(u-v) d x\right| \\
& \quad \leq \int_{\Omega \backslash \Omega_{\lambda}} a(x)\left|\left(|\nabla u|^{p-2} \nabla u-|\nabla v|^{p-2} \nabla v\right) \cdot \nabla \phi_{\lambda}(x) S_{\eta}(u-v)\right| d x \\
& \quad \leq \int_{\Omega \backslash \Omega_{\lambda}} a(x)\left|\left(|\nabla u|^{p-2} \nabla u-|\nabla v|^{p-2} \nabla v\right)\right|\left|\nabla \phi_{\lambda}(x)\right| d x \\
& \quad=\frac{1}{\lambda} \int_{\Omega \backslash \Omega_{\lambda}} a(x)\left|\left(|\nabla u|^{p-2} \nabla u-|\nabla v|^{p-2} \nabla v\right)\right||\nabla a(x)| d x
\end{aligned}
$$


Since (1.14), for small enough $\lambda>0$,

$$
\frac{1}{\lambda}\left(\lambda \int_{\Omega \backslash \Omega_{\lambda}}|\nabla a|^{p} d x\right)^{\frac{1}{p}} \leq c
$$

using the Hölder inequality, one has

$$
\begin{aligned}
& \left|\int_{\Omega} a(x)\left(|\nabla u|^{p-2} \nabla u-|\nabla v|^{p-2} \nabla v\right) \cdot \nabla \phi_{\lambda}(x) S_{\eta}(u-v) d x\right| \\
& \leq \frac{c}{\lambda}\left[\int_{\Omega \backslash \Omega_{\lambda}} a(x)|\nabla u|^{p-1}|\nabla a| d x+\int_{\tau}^{s} \int_{\Omega \backslash \Omega_{\lambda}} a(x)|\nabla v|^{p-1}|\nabla a| d x\right] \\
& \leq \frac{c}{\lambda}\left(\int_{\Omega \backslash \Omega_{\lambda}} a|\nabla a|^{p} d x\right)^{\frac{1}{p}}\left(\int_{\Omega \backslash \Omega_{\lambda}} a(x)|\nabla u|^{p} d x\right)^{\frac{p-1}{p}} \\
& \quad+\frac{c}{\lambda}\left(\int_{\Omega \backslash \Omega_{\lambda}} a(x)|\nabla a|^{p} d x\right)^{\frac{1}{p}}\left(\int_{\Omega \backslash \Omega_{\lambda}} a(x)|\nabla v|^{p} d x\right)^{\frac{p-1}{p}} \\
& \leq c\left(\int_{\Omega \backslash \Omega_{\lambda}} a(x)|\nabla u|^{p} d x\right)^{\frac{p-1}{p}}+c\left(\int_{\Omega \backslash \Omega_{\lambda}} a(x)|\nabla v|^{p} d x\right)^{\frac{p-1}{p}} .
\end{aligned}
$$

Then

$$
\lim _{\lambda \rightarrow 0} \lim _{\eta \rightarrow 0}\left|\int_{\Omega} a(x)\left(|\nabla u|^{p-2} \nabla u-|\nabla v|^{p-2} \nabla v\right) \cdot \nabla \phi_{\lambda}(x) S_{\eta}(u-v) d x\right|=0 .
$$

Secondly, for any given $\lambda>0$, since $a(x)>0, x \in \Omega$ and $|\nabla u| \in L_{\text {loc }}^{p}(\Omega)$, for the first part of the fourth term on the left hand side of (4.3), one has

$$
\left|\phi_{\lambda}\left[b_{i}(u, x, t)-b_{i}(v, x, t)\right] S_{\eta}^{\prime}(u-v)(u-v)_{x_{i}}\right| \leq c(\lambda)|u-v| S_{\eta}^{\prime}(u-v)\left|(u-v)_{x_{i}}\right|,
$$

by the Lebesgue dominated convergence theorem, by (3.2), one has

$$
\lim _{\eta \rightarrow 0}\left|\int_{\Omega} \phi_{\lambda}\left[b_{i}(u, x, t)-b_{i}(v, x, t)\right] S_{\eta}^{\prime}(u-v)(u-v)_{x_{i}} d x\right|=0
$$

Then

$$
\lim _{\lambda \rightarrow 0} \lim _{\eta \rightarrow 0}\left|\int_{\Omega} \phi_{\lambda}\left[b_{i}(u, x, t)-b_{i}(v, x, t)\right] S_{\eta}^{\prime}(u-v)(u-v)_{x_{i}} d x\right|=0 .
$$

Once more, for the second part of the fourth term on the left hand side of (4.3), one can deal with it in two cases according to the conditions (1.15) and (1.16), respectively. In detail, if $|\nabla a|=0, x \in \partial \Omega$, then

$$
\begin{aligned}
& \lim _{\lambda \rightarrow 0} \lim _{\eta \rightarrow 0}\left|\int_{\Omega} \phi_{\lambda x_{i}}\left[b_{i}(u, x, t)-b_{i}(v, x, t)\right] S_{\eta}(u-v) d x\right| \\
& \quad \leq c \lim _{\lambda \rightarrow 0} \int_{\Omega}\left|\phi_{\lambda x_{i}}\left[b_{i}(u, x, t)-b_{i}(v, x, t)\right]\right| d x \\
& \quad \leq \lim _{\lambda \rightarrow 0} \frac{c}{\lambda} \int_{\Omega \backslash \Omega_{\lambda}}|\nabla a| d x
\end{aligned}
$$




$$
\begin{aligned}
& =c \int_{\partial \Omega}|\nabla a| d \sigma \\
& =0 .
\end{aligned}
$$

If (1.16) is true, when $x \in \partial \Omega, b_{i}(\cdot, x, t)=0$ is reasonable (we would like to suggest that we do not require $\left.u\right|_{x \in \partial \Omega}=0$ here), then

$$
\begin{aligned}
& \lim _{\lambda \rightarrow 0} \lim _{\eta \rightarrow 0}\left|\int_{\Omega} \phi_{\lambda x_{i}}\left[b_{i}(u, x, t)-b_{i}(v, x, t)\right] S_{\eta}(u-v) d x\right| \\
& \quad \leq c \lim _{\lambda \rightarrow 0} \int_{\Omega}\left|\phi_{\lambda x_{i}}\left[b_{i}(u, x, t)-b_{i}(v, x, t)\right]\right| d x \\
& \quad \leq \lim _{\lambda \rightarrow 0} \frac{c}{\lambda} \int_{\Omega \backslash \Omega_{\lambda}}\left|b_{i}(u, x, t)-b_{i}(v, x, t)\right| d x \\
& \quad=c \int_{\partial \Omega}\left|b_{i}(u, x, t)-b_{i}(v, x, t)\right| d \sigma \\
& \quad=0 .
\end{aligned}
$$

Last but not least, for the first term of the left term of (4.3), one has

$$
\begin{aligned}
& \lim _{\eta \rightarrow 0} \lim _{\lambda \rightarrow 0} \int_{\tau}^{s} \int_{\Omega} \phi_{\lambda}(x) S_{\eta}(u-v) \frac{\partial\left(|u|^{\beta-1} u-|v|^{\beta-1} v\right)}{\partial t} d x d t \\
& \quad=\lim _{\eta \rightarrow 0} \int_{\tau}^{s} \int_{\Omega} S_{\eta}(u-v) \frac{\partial\left(|u|^{\beta-1} u-|v|^{\beta-1} v\right)}{\partial t} d x d t \\
& \quad=\int_{\tau}^{s} \int_{\Omega} \operatorname{sign}(u-v) \frac{\partial\left(|u|^{\beta-1} u-|v|^{\beta-1} v\right)}{\partial t} d x d t \\
& \quad=\int_{\tau}^{s} \int_{\Omega} \operatorname{sign}\left(|u|^{\beta-1} u-|v|^{\beta-1} v\right) \frac{\partial\left(|u|^{\beta-1} u-|v|^{\beta-1} v\right)}{\partial t} d x d t \\
& \quad=\int_{\tau}^{s} \frac{d}{d t}\left\||u|^{\beta-1} u-|v|^{\beta-1} v\right\|_{L^{1}(\Omega)} d t .
\end{aligned}
$$

After letting $\lambda \rightarrow 0$, let $\eta \rightarrow 0$ in (4.3). By (4.4), (4.6)-(4.11), one has

$$
\begin{aligned}
& \left.\int_{\Omega}|| u\right|^{\beta-1} u(x, t)-|v|^{\beta-1} v(x, t) \mid d x \\
& \quad \leq\left.\int_{\Omega}|| u_{0}\right|^{\beta-1} u_{0}(x)-\left|v_{0}\right|^{\beta-1} v_{0}(x) \mid d x .
\end{aligned}
$$

By a minor modification of the above proof, we can prove Theorem 1.6.

Proof of Theorem 1.6 Since $\int_{\Omega} a(x)^{-\frac{1}{p-1}} d x<\infty$, one can define the trace of weak solution $u$ on the boundary. Accordingly, the partial boundary value condition (1.23) is reasonable. Now,

$$
\begin{aligned}
& \lim _{\lambda \rightarrow 0}\left|\int_{\Omega} \phi_{\lambda x_{i}}\left[b_{i}(u, x, t)-b_{i}(v, x, t)\right] S_{\eta}(u-v) d x\right| \\
& \quad \leq \lim _{\lambda \rightarrow 0} \frac{c}{\lambda} \int_{\Omega \backslash \Omega_{\lambda}}\left|h_{i}(x) a_{x_{i}}\right||u-v| d x
\end{aligned}
$$




$$
\begin{aligned}
& =c \int_{\partial \Omega}|u-v|\left|h_{i}(x) a_{x_{i}}\right| d \sigma \\
& =0 .
\end{aligned}
$$

The rest of the proof Theorem 1.6 can be completed just in the same way as that of Theorem 1.4.

From the proofs of Theorem 1.4 and Theorem 1.6, we easily have the following corollary, no matter whether $\int_{\Omega} a^{-\frac{1}{p-1}}(x) d x<\infty$ or not.

Corollary 4.1 Let $u(x, t)$ and $v(x, t)$ be two solutions of Eq. (1.9) with the initial values $u_{0}(x)$ and $v_{0}(x)$, respectively, but without any boundary value condition. If $a(x)$ satisfies (1.14), $b_{i}(\cdot, x, t)$ satisfies $(1.22)$, and

$$
|\nabla a| h_{i}(x)=0, \quad i=1,2, \ldots, N, x \in \partial \Omega,
$$

then the stability of weak solutions is true in the sense of (1.13).

\section{The Proof of Theorem 1.5}

From (2.16), we see that (1.6) is equivalent to

$$
\iint_{Q_{T}}\left(\frac{\partial u}{\partial t} \varphi+\frac{a(x)}{m^{p-1}}\left|\nabla u^{m}\right|^{p-2} \nabla u^{m} \cdot \nabla \varphi+\sum_{i=1}^{N} a_{i}(u, x, t) \varphi_{x_{i}}\right) d x d t=0,
$$

with $m=1+\frac{\alpha}{p-1}$.

Proof of Theorem 1.5 Let $u(x, t), v(x, t)$ be two solutions of Eq. (1.1) with the initial values $u_{0}(x), v_{0}(x)$. By taking the limit, one can choose $S_{\eta}\left(a\left(u^{m}-v^{m}\right)\right)$ as the test function;

$$
\begin{aligned}
\int_{\Omega} & S_{\eta}\left(a\left(u^{m}-v^{m}\right)\right) \frac{\partial(u-v)}{\partial t} d x \\
& +\frac{1}{m^{p-1}} \int_{\Omega} a^{2}(x)\left(\left|\nabla u^{m}\right|^{p-2} \nabla u^{m}-\left|\nabla v^{m}\right|^{p-2} \nabla v^{m}\right) \\
& +\nabla\left(u^{m}-v^{m}\right) S_{\eta}^{\prime}\left(a\left(u^{m}-v^{m}\right)\right) d x \\
& +\int_{\Omega} a(x)\left(\left|\nabla u^{m}\right|^{p-2} \nabla u^{m}-\left|\nabla v^{m}\right|^{p-2} \nabla v^{m}\right) \cdot \nabla a\left(u^{m}-v^{m}\right) S_{\eta}^{\prime}\left(a\left(u^{m}-v^{m}\right)\right) d x \\
& +\int_{\Omega}\left[a_{i}(u, x, t)-a_{i}(v, x, t)\right] S_{\eta}^{\prime}\left(a\left(u^{m}-v^{m}\right)\right)\left[a_{x_{i}}^{\beta}\left(u^{m}-v^{m}\right)+a\left(u^{m}-v^{m}\right)_{x_{i}}\right] d x \\
= & 0 .
\end{aligned}
$$

As before, one has

$$
\begin{aligned}
& \lim _{\eta \rightarrow 0} \int_{\Omega} S_{\eta}\left(a\left(u^{m}-v^{m}\right)\right) \frac{\partial(u-v)}{\partial t} d x=\frac{d}{d t} \int_{\Omega}|u-v| d x, \\
& \int_{\Omega} a^{2}(x)\left(\left|\nabla u^{m}\right|^{p-2} \nabla u^{m}-\left|\nabla v^{m}\right|^{p-2} \nabla v^{m}\right) \cdot \nabla\left(u^{m}-v^{m}\right) S_{\eta}^{\prime}\left(a\left(u^{m}-v^{m}\right)\right) d x \geq 0 .
\end{aligned}
$$


Chan and Ouyang Journal of Inequalities and Applications

(2019) 2019:235

Page 15 of 17

Since $|\nabla a(x)| \leq c$ in $\Omega$, one has

$$
\begin{aligned}
& \left|\int_{\Omega} a(x)\left(u^{m}-v^{m}\right) S_{\eta}^{\prime}\left(a\left(u^{m}-v^{m}\right)\right)\left(\left|\nabla u^{m}\right|^{p-2} \nabla u^{m}-\left|\nabla v^{m}\right|^{p-2} \nabla v^{m}\right) \cdot \nabla a d x\right| \\
& \quad \leq c \int_{\Omega}\left|a\left(u^{m}-v^{m}\right) S_{\eta}^{\prime}\left(a\left(u^{m}-v^{m}\right)\right)\left(\left|\nabla u^{m}\right|^{p-2} \nabla u^{m}-\left|\nabla v^{m}\right|^{p-2} \nabla v^{m}\right)\right| d x
\end{aligned}
$$

and

$$
\begin{aligned}
\int_{\Omega}\left|a\left(u^{m}-v^{m}\right) S_{\eta}^{\prime}\left(a\left(u^{m}-v^{m}\right)\right)\left(\left|\nabla u^{m}\right|^{p-2} \nabla u^{m}-\left|\nabla v^{m}\right|^{p-2} \nabla v^{m}\right)\right| d x \\
=\int_{\left\{\Omega: a\left|u^{m}-v^{m}\right|<\eta\right\}} \mid a^{-\frac{p-1}{p}} a\left(u^{m}-v^{m}\right) S_{\eta}^{\prime}\left(a\left(u^{m}-v^{m}\right)\right) \\
\cdot a^{\frac{p-1}{p}}\left(\left|\nabla u^{m}\right|^{p-2} \nabla u^{m}-\left|\nabla v^{m}\right|^{p-2} \nabla v^{m}\right) \mid d x \\
\leq\left(\int_{\{\Omega: a|u-v|<\eta\}}\left|a^{-\frac{p-1}{p}} a\left(u^{m}-v^{m}\right) S_{\eta}^{\prime}\left(a\left(u^{m}-v^{m}\right)\right)\right|^{p} d x\right)^{\frac{1}{p}} \\
\quad\left(\int_{\{\Omega: a|u-v|<\eta\}} a(x)\left(\left|\nabla u^{m}\right|^{p}+\left|\nabla v^{m}\right|^{p}\right) d x\right)^{\frac{p-1}{p}} \cdot
\end{aligned}
$$

If $\left\{x \in \Omega: u^{m}-v^{m}=0\right\}$ has 0 measure, since (1.17)

$$
\int_{\Omega} a(x)^{-(p-1)} d x<\infty
$$

one has

$$
\int_{\left\{\Omega: a\left|u^{m}-v^{m}\right|<\eta\right\}}\left|a^{-\frac{p-1}{p}} a\left(u^{m}-v^{m}\right) S_{\eta}^{\prime}\left(a\left(u^{m}-v^{m}\right)\right)\right|^{p} d x<\infty
$$

and

$$
\begin{aligned}
& \lim _{\eta \rightarrow 0}\left(\int_{\left\{\Omega: a\left|u^{m}-v^{m}\right|<\eta\right\}} a(x)\left(\left|\nabla u^{m}\right|^{p}+\left|\nabla v^{m}\right|^{p}\right) d x\right)^{\frac{p-1}{p}} \\
& \quad=\left(\int_{\left\{\Omega:\left|u^{m}-v^{m}\right|=0\right\}} a(x)\left(\left|\nabla u^{m}\right|^{p}+\left|\nabla v^{m}\right|^{p}\right) d x\right)^{\frac{p-1}{p}} \\
& \quad=0 .
\end{aligned}
$$

If $\left\{x \in \Omega: u^{m}-v^{m}=0\right\}$ has a positive measure, obviously,

$$
\begin{aligned}
& \lim _{\eta \rightarrow 0}\left(\int_{\left\{\Omega: a\left|u^{m}-v^{m}\right|<\eta\right\}}\left|a^{-\frac{p-1}{p}} a\left(u^{m}-v^{m}\right) S_{\eta}^{\prime}\left(a\left(u^{m}-v^{m}\right)\right)\right|^{p} d x\right)^{\frac{1}{p}} \\
& \quad=\left(\int_{\left\{\Omega:\left|u^{m}-v^{m}\right|=0\right\}}\left|a^{-\frac{p-1}{p}} a\left(u^{m}-v^{m}\right) S_{\eta}^{\prime}\left(a\left(u^{m}-v^{m}\right)\right)\right|^{p} d x\right)^{\frac{1}{p}} \\
& \quad=0 .
\end{aligned}
$$


In both cases, using Lebesgue's dominated convergence theorem, by (3.2), one has

$$
\lim _{\eta \rightarrow 0}\left|\int_{\Omega} a\left(u^{m}-v^{m}\right) S_{\eta}^{\prime}\left(a\left(u^{m}-v^{m}\right)\right)\left(\left|\nabla u^{m}\right|^{p-2} \nabla u^{m}-\left|\nabla v^{m}\right|^{p-2} \nabla v^{m}\right) d x\right|=0 .
$$

In addition, by (1.19) and (1.20), one has

$$
\begin{aligned}
& \left|\int_{\Omega}\left[a_{i}(u, x, t)-a_{i}(v, x, t)\right] a_{x_{i}}\left(u^{m}-v^{m}\right) S_{\eta}^{\prime}\left(a\left(u^{m}-v^{m}\right)\right) d x\right| \\
& \quad \leq c \int_{\Omega} g_{i}(x) \frac{|\nabla a|}{a} a\left(u^{m}-v^{m}\right) S_{\eta}^{\prime}\left(a\left(u^{m}-v^{m}\right)\right) d x \\
& \quad \rightarrow 0
\end{aligned}
$$

as $\eta \rightarrow 0$.

Moreover, condition (1.18) yields

$$
\begin{aligned}
& \left|\int_{\Omega}\left[a_{i}(u, x, t)-a_{i}(v, x, t)\right] a\left(u^{m}-v^{m}\right)_{x_{i}} S_{\eta}^{\prime}\left(a\left(u^{m}-v^{m}\right)\right) d x\right| \\
& \quad=\left|\int_{\Omega} a^{1-\frac{1}{p}}\left[a_{i}(u, x, t)-a_{i}(v, x, t)\right] S_{\eta}^{\prime}\left(a\left(u^{m}-v^{m}\right)\right) a^{-\frac{1}{p}}\left(u^{m}-v^{m}\right)_{x_{i}} d x\right| \\
& \quad \leq c\left(\int_{\Omega}\left|a\left(u^{m}-v^{m}\right) S_{\eta}^{\prime}\left(a\left(u^{m}-v^{m}\right)\right)\right|^{\frac{p}{p-1}} d x\right)^{\frac{p-1}{p}}\left(\int_{\Omega} a(x)\left(\left|\nabla u^{m}\right|^{p}+\left|\nabla v^{m}\right|\right)\right)^{\frac{1}{p}} \\
& \quad \rightarrow 0,
\end{aligned}
$$

as $\eta \rightarrow 0$.

Let $\eta \rightarrow 0$ in (5.2). By (5.3)-(5.11), one has

$$
\int_{\Omega}|u(x, t)-v(x, t)| \mathrm{d} x \leq c \int_{\Omega}\left|u_{0}(x)-v_{0}(x)\right| \mathrm{d} x, \quad \forall t \in[0, T) .
$$

Theorem 1.5 is proved.

\section{Acknowledgements}

The authors would like to thank everyone for help.

\section{Funding}

The paper is supported by Natural Science Foundation of Fujian province (no: 2019J01858), supported by Science Foundation of Xiamen University of Technology, China.

\section{Availability of data and materials}

No applicable.

Competing interests

The authors declare that they have no competing interests.

\section{Authors' contributions}

The authors read and approve the final manuscript.

\section{Publisher's Note}

Springer Nature remains neutral with regard to jurisdictional claims in published maps and institutional affiliations. Received: 19 April 2019 Accepted: 26 August 2019 Published online: 02 September 2019 


\section{References}

1. Aris, R.: In: The Mathematical Theory of Diffusion and Reaction in Permeable Catalysts, I, II. Clarendon, Oxford (1975)

2. Bear, J.: Dynamics of Fluids in Porous Media. American Elsevier, New York (1972)

3. Childs, E.C.: An Introduction to the Physical Basis of Soil Water Phenomena. Wiley, London (1969)

4. Richards, L.A.: Capillary conduction of liquids through porous mediums. Physics 1, 318-333 (1931)

5. Gilding, B.G., Peletier, L.A.: The Cauchy problem for an equation in the theory of infiltration. Arch. Rational Mech. Anal. $61,127-140(1976)$

6. Zhao, J., Yuan, H.: The Cauchy problem of some doubly nonlinear degenerate parabolic equations. Chinese Anal. Math., Ser A. 16(2), 179-194 (1995) (in Chinese)

7. Fan, $\mathrm{H}$.: Cauchy problem of some doubly degenerate parabolic equations with initial datum a measure. Acta Math. Sinica, Ser. B 20(4), 663-682 (2004)

8. Shang, H.: Doubly nonlinear parabolic equations with measure data. Ann. Mat. Pura Appl. 192, $273-296$ (2013)

9. Otto, F.: $L^{1}$-Contraction and uniqueness for quasilinear elliptic parabolic equations. J. Differential Equations 131 20-38 (1996)

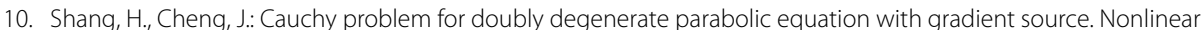
Anal. 113, 323-338 (2015)

11. Lu, G.: Nonlinear degenerate parabolic equations in infiltration through a porous medium. Comm. Nonlinear Sci. Num. Simu. 3, 97-100 (1998)

12. Ohara, Y.: $L^{\infty}$ estimates of solutions of some nonlinear degenerate parabolic equations. Nonlinear Anal. 18, 413-426 (1992)

13. Chen, C., Wang, R.: Global existence and $L^{\infty}$ estimates of solution for doubly degenerate parabolic equation. Acta Math. Sinica, Ser A. 44, 1089-1098 (2001) (in Chinese)

14. Andreucci, D., Cirmi, G.R., Leonardi, S., Tedeev, A.F.: Large time behavior of solutions to the Neumann problem for a quasilinear second order degenerate parabolic equation in domains with noncompact boundary. J. Differential Equations 174, 253-288 (2001)

15. Manfredi, J., Vespri, V.: Large time behavior of solutions to a class of doubly nonlinear parabolic equations. Electronic J. Differential Equations 1994(2), 1-16 (1994)

16. Lee, K., Petrosyan, A., Vázquez, J.L.: Large time geometric properties of solutions of the evolution $p$-Laplacian equation. J. Differential Equations 229, 389-411 (2006)

17. Yuan, J., Lian, Z., Cao, L., Gao, J., Xu, J.: Extinction and positivity for a doubly nonlinear degenerate parabolic equation. Acta Math. Sinica, Ser. B 23, 1751-1756 (2007)

18. Tedeev, A.F.: The interface blow-up phenomenon and local estimates for doubly degenerate parabolic equations Appl. Anal. 86(6), 755-782 (2007)

19. Ye, H., Yin, J.: Propagation profile for a non-Newtonian polytropic filtration equation with orientated convection. J. Math. Anal. Appl. 421, 1225-1237 (2015)

20. Wu, Z., Zhao, J., Yin, J., Li, H.: Nonlinear Diffusion Equations. Word Scientific Publishing, Singapore (2001)

21. Zhou, Z., Guo, Z., Wu, B.: A doubly degenerate diffusion equation in multiplicative noise removal models. J. Math. Anal. Appl. 458, 58-70 (2018)

22. Suna, J., Yin, J., Wang, Y.: Asymptotic bounds of solutions for a periodic doubly degenerate parabolic equation. Nonlinear Anal. 74, 2415-2424 (2011)

23. Gianni, R., Tedeev, A., Vespri, V.: Asymptotic expansion of solutions to the Cauchy problem for doubly degenerate parabolic c equations with measurable coefficients. Nonlinear Anal. 138, 111-126 (2016)

24. Droniou, J., Eymard, R., Talbot, K.S.: Convergence in $C\left([0, T] ; L^{2}(\Omega)\right)$ of weak solutions to perturbed doubly degenerate parabolic equations. J. Differential Equations 260, 7821-7860 (2016)

25. Zou, W., Li, J.: Existence and uniqueness of solutions for a class of doubly degenerate parabolic equations. J. Math. Anal. Appl. 446, 1833-1862 (2017)

26. Li, Q.: Weak Harnack estimates for supersolutions to doubly degenerate parabolic equations. Nonlinear Anal. 170 88-122 (2018)

27. Yin, J., Wang, C.: Properties of the boundary flux of a singular diffusion process. Chinese Ann. Math. Ser. B 25B(2), 175-182 (2004)

28. Yin, J., Wang, C.: Evolutionary weighted $p$-Laplacian with boundary degeneracy. J. Differential Equations 237, 421-445 (2007)

29. Zhan, H.: The uniqueness of a nonlinear diffusion equation related to the $p$-Laplacian. J. Inequal. Appl. 2018(7) (2018)

30. Zhan, H.: Infiltration equation with degeneracy on the boundary. Acta Appl. Math. 153(1), 147-161 (2018)

31. Zhan, H.: Solutions to polytropic filtration equations with a convection term. Electron. J. Differ. Equ. $2017,207(2017)$

32. Simon, J.: Compact sets in the space $L^{P}(0, t ; B)$. Ann. Mat. Pura Appl. 146, 65-96 (1987)

33. Zhao, J.: Existence and nonexistence of solutions for $u_{t}=\operatorname{div}\left(|\nabla u|^{p-2} \nabla u\right)+f(\nabla u, u, x, t)$. J. Math. Anal. Appl. 172, 130-146 (1993)

34. Li, Z., Yan, B., Gao, W.: Existence of solutions to a parabolic $p(x)$-Laplace equation with convection term via $L^{1}$-estimates. Electron. J. Differ. Equ. 2014, 46 (2014) 